\title{
BIM ON ARTIFICIAL INTELLIGENCE FOR DECISION SUPPORT IN E-HEALTH
}

\author{
B. Plaß *, C. Prudhomme, J. J. Ponciano \\ i3mainz, Institute for Spatial Information and Surveying Technology, Mainz University of Applied Sciences, Germany - \\ (bastian.plass, claire.prudhomme, jean-jacques.ponciano)@hs-mainz.de
}

\author{
Commission II, WG II/3
}

KEY WORDS: Decision Support System, Knowledge Base, Building Information Modeling, Artificial Intelligence, Care 4.0, Point Cloud, Semantic Segmentation, Object Detection.

\begin{abstract}
:
Looking ahead of 2070, the number of the elderly population will increase rapidly in the European Union and beyond. As society ages, it will be confronted to novel challenges related with other concerns like the concept aging in place that the majority of the elderly prefer. Concerning that, the living space must be adapted to the requirements of people with a disability, to support their relatives or friends that will become more and more important in future due to a lack of professional's and both overstressed and expensive hospitals or nursing homes. Compounding this, those living space requirements are highly individual, depending on the disease. Our study focuses on a medical white box decision support system providing advice even for unknowledgeable users by evaluating the suitability of an elderly's living environment in terms of their individual disease. In this paper, we propose tackling this issue with a decision support system linked to Building Information Modeling (BIM) and based on Artificial Intelligence using semantic technologies. The proposed approach's contribution is a reliable process that uses up-to-date 3D point cloud data of the person's living environment and predicts suitable, non-suitable and adaptable zones therein according to different pathologies using formalised knowledge. We are able to provide deep expert knowledge linked from different domains inside a knowledge base and thus produce an outcome through BIM, which is understandable and helpful for two types of users, ordinary people concerned by the matter and building experts. We illustrate our methodology by a proof of concept concerning a wheel-chaired person.
\end{abstract}

\section{INTRODUCTION}

The European Union is facing a variety of challenges in the future as the changing demographic structure. As a result, the number of the elderly population will increase due to arising life expectancy and dropping birth rates by 2070 (European Commission 2020). Relating to this long-term trend, medical and social investigations identified the demand of senior citizens concerning their future life even under care. The majority of the elderly prefer an independent and self-determined life in a familiar environment (Harrefors et al. 2009; Stula 2012), both in urban and rural areas (Ohta et al. 2020). Thus, the concept aging in place ${ }^{1}$ became an advantage in terms of autonomy, safety, familiarity and the elderly's identity (Wiles et al. 2012). However, this only takes into account the fragile situation of the European healthcare system partially. Often, aging is related to the appearance of diseases that need to be treated. Having in need of care concerned elderlies, hereinafter patients, remain in their homes as long as possible, care services needs to be provided in a distributed manner as well, despite already overburdened healthcare system and high workloads. To turn these challenges into opportunities digital transformation helping healthcare system to improve services and treatments.

E-Health is an emerging way to deliver healthcare through applications using information and communication technology (ICT), like personal health (PH) or assisted living (AL) systems for the elderly or disabled (Avila and Sampogna 2011). Particularly in the latter domain, current technical efforts aim at treatment and monitoring of patients as well as assistance to care professionals (Rösler et al. 2018; Banbury et al. 2014). A key technology concerning AL are decision support systems (DSS) that are usually associated with processes that aim to support people in making decisions by collecting and determining relevant information (Papathanasiou et al. 2016). As a subject of intensive research for years, DSS have already been successfully used in various fields. Today's market offers many examples in common day-to-day usage to improve decision-making in biology (Suphavilai et al. 2018; Fiannaca et al. 2011), economics (Dutta et al. 2011), engineering (Simões-Marques 2016; Papathoma-Köhle et al. 2019; Coelho et al. 2021) or e-commerce (Leung et al. 2018; Masaro et al. 2020). Refer to Sutton et al. (2020) and Stark et al. (2019), DSS are also widely used in the medical domain for disease diagnostic (Kumar et al. 2011; Kihlgren et al. 2016) and patient monitoring (Kaczmarek et al. 2011; Billis et al. 2015; Hussain et al. 2015). In general, studies are patient-related and assist both at clinical sites or in their living environment. So called medical DSS (MDSS) produce recommendations to the caregiver based on recognition of patients habits or anomalies. Often, just a certain use case such as Alzheimer disease is considered. The elderly that want to age in place, usually tend to suffer multiple diseases that cause new examinations whether safe living at home is still possible.

Our research provides a solution based on DSS to address the dynamics of aging in place in terms of changing domestic preconditions as a result of various disease profiles. To achieve this, multiple technologies such as Building Information Modeling (BIM) and Artificial Intelligence (AI) are utilized to create a system, which is able to provide insightful information about an as-is home of a senior citizen with already existing disabilities. With respect to the patient's disease profile, our MDSS evaluates the suitability of the living space and gives advice on necessary home modifications, improving the living quality and safety. As main users patients or relatives are addressed in order to discharge the healthcare system. In humanrelated MDSS applications it is urgent to justify each decision in a transparent and comprehensive manner (Richter and Vogel

\footnotetext{
* Corresponding author

Ability to live in one's own home regardless of age or disability.
} 
2020). This is why we chose a knowledge-based approach already suggested in similar domain by Bouchard et al. (2007), Kaczmarek et al. (2011), Augustyniak (2013), Billis et al. (2015), Chiang and Liang (2015), Hussain et al. (2015) and Lopez-Guede et al. (2015).

As a proof of concept of our approach we present in this paper the case study of a wheel-chaired person. In order to evaluate the suitability of the living environment, up-to-date information is needed as well as criteria, which can be checked. According to state of the art, geometric information for 3D representation can be obtained from point cloud data acquired by scanning or imaging techniques. Based on point cloud data, geometric representations with semantic attributes of constituent interiors such as walls, doors, stairs, tables or chairs need to be modeled and stored as component-related into a graphical BIM database intending to provide information about constitute elements with its functionality and suitability for a wheel-chaired person. Geometric information from those solid 3D building components contribute to the transparency of the decisions made by our approach and thus plays a major role for the main users. Beyond that, the knowledge for evaluation purposes need to be formalised as an ontology. Four main components are considered in our knowledge-based approach such as (a) the disease profile of a wheel-chaired according to barrier-free living requirements under the terms of DIN 18040-2, (b) the data processing aside from (c) the expert knowledge in order to determine the suitability of a living environment by logical linkage of the states for each interior element which are (d) finally transferred into a BIM model. For reasoning in the penultimate aspect, a deeper 3D understanding beyond the elementwise BIM provision is needed and realised thanks to KnowDIP (Ponciano et al. 2017). Those fine BIM components are checked against the formulised expert content, resulting in the living environment evaluation concerning criteria of barrier-free living and unrestricted wheelchair usage. A more precise description of the knowledgebased approach is described in the methodology. Further information concerning our chosen case study is given in the proof of concept section. We transfer standardised expert knowledge from professional domains into reusable ontologies and thus create an extensible approach for transparent suitability evaluations of a patient's living environments. With our knowledge-based DSS, we expand the portfolio of E-Health technology and contribute to sustain life quality, autonomy and safety of patients aging in place for a longer period of time.

\section{RELATED WORK AND CHALLENGES}

During our studies, we encountered several challenges to conceptualize the proposed approach. As a conclusion, those can be subdivided into four areas.

\subsection{MDSS requirements}

In spite of an arising application domain, there is a lack of recent research in the field of MDSS for homecare describing requirements and study experiences. As reviewed by Marschollek (2012), Baumgärtel et al. (2018) and Stark et al. (2019), only a few papers offer details about the knowledge base (KB), system architecture and study outcome. In addition, presented researches lack of accuracy, best practices, comprehensibility, interchangeability, user acceptance, stability and insightful evaluation methods (Richter and Vogel 2020; Baumgärtel et al. 2018; Baig et al. 2019; Manar et al. 2017; Stark et al. 2019). Considering this, we focus on a case research based on expert rules to reason of a suitable living environment according to different patient's diseases. We tackle the lack of evidence and accuracy by a MDSS linked to BIM and based on
AI using semantic technologies to represent extensible knowledge and perform insightful understanding of as-is home scene. As already stated in literature research, our proof of concept also lacks evaluation methods. However, this will be addressed in a future real life study.

\subsection{Automatic 3D understanding and data processing}

As already mentioned above, accurate 3D data are highly important for obtaining 3D representation from objects such as in living environmental sites. Scanning (Lehtola et al. 2017; Bassier et al. 2015; Lercari 2016) or imaging (Lin et al. 2017; Lu and Lee 2017) methods provide dense point cloud data much faster than in-situ measurements and thus are considered as state of the art acquisition techniques by numerous literature. Compared to mostly efficient and fast data acquisition, the processing of the dense $3 \mathrm{D}$ point cloud is a complex and challenging task. The automatic understanding of unstructured 3D point cloud data has become a popular research field in recent years that is not only limited to BIM in architecture, engineering and construction (AEC) industry. Methods such as semantic segmentation and 3D object detection can subdivide a point cloud scene into its constituent elements in order to gain a deeper understanding and thus help in decision-making. Research relating these methods in 3D data has gained high impact factor, shown by the appearance of well-known datasets like ModelNet (Wu et al. 2015) and ShapeNet (Chang et al. 2015) for recognition of Computer-Aided Design (CAD) models or for building and furniture elements by Stanford Large-Scale Indoor Spaces (S3DIS) (Armeni et al. 2016). Recent research substitutes rule-based methods like random sample consensus (RANSAC) or Hough transform (Adan and Huber 2011; Jung et al. 2016) for simple geometry detection and has been dedicated in using computer vision and deep learning approaches to train models end-to-end for direct mapping from 3D inputs to object labels (Chen et al. 2019; Malinverni et al. 2019; Qi et al. 2016; Ben-Shabat et al. 2018). However, these approaches still remain inflexible and unable to detect objects or geometry for which they are not trained. Furthermore, just a small change of the data acquisition process or external factors that influence the $3 \mathrm{D}$ data can result in a change that turns the elaborately trained model useless. Point cloud data from living environments are characterised by unstructured, scattered and spatiotemporal objects (Chen et al. 2019; Bassier et al. 2019). Face to such characteristics, the object detection is currently a challenging task despite deep models due to lack of training data. On the contrary, other approaches based on semantic technologies propose to use prior knowledge about object, point cloud and sensing process to drive and adapt the data processing (Poux et al. 2018; Dietenbeck et al. 2017; Poux and Billen 2019). These methods bring a huge flexibility to process $3 \mathrm{D}$ point clouds based on less strict acquisition criteria and are able to outperform deep learning accuracy. For this reason we build our method on a knowledge-based approach using AI on semantic technologies to understand $3 \mathrm{D}$ data in a more comprehensive and accurate way (Poux and Ponciano 2020).

\subsection{On-site construction management}

The patient's living environment constitutes the main data component for evaluating the suitability to aging in place concerning a disease profile. As an individual candidate, this data represents an exchangeable value and acts as the information backbone of our knowledge-based MDSS. Discretised as an unstructured but colorized 3D point cloud, the as-is living site provide all valuable geometric and semantic data that need to be modeled and stored as component-related into a database. A solution to organize this data is given by the most advanced 


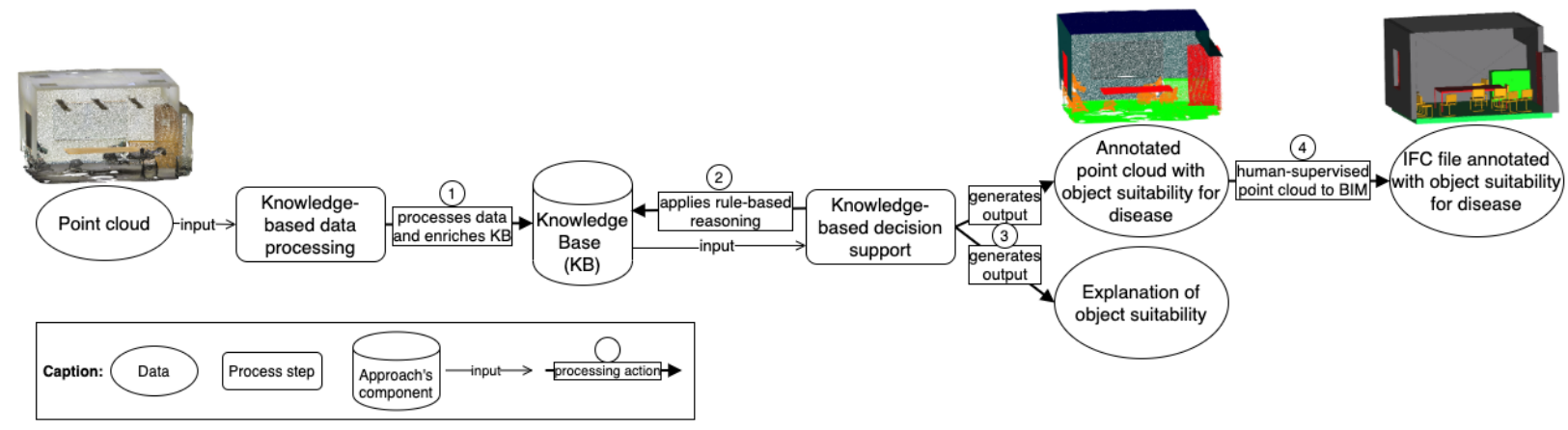

Figure 1. Overview of the proposed methodology.

method of geometric modeling in recent years: Building Information Modeling (BIM). The concept of BIM is manifold in AEC industry. In general, it represents a digital facility as a shared information resource providing a reliable basis for various stakeholders for decision-making processes during its life cycle (Borrmann et al. 2015; Eastman 2008). Within our study we use the term as a comprehensive object-oriented 3D building model with semantic, topologic and geometric attributes. As a graphical 3D database, information related to building components are stored and provided through Industry Foundation Class (IFC) description in solid model paradigm. IFC is an open, international standard (ISO 16739-1) for interdisciplinary collaboration purposes providing agnostic and interoperable capabilities across a broad range of software platforms for lots of different use cases. Intended to experts in the vast AEC industry, the $3 \mathrm{D}$ data schema granularity is fine and due to multiple redundancies even for small building sites the information in such data is too heavy to be treated automatically. This is why we have added a manual step that uses the annotated point cloud resulting from our approach to generate an IFC file enriched with the suitability states of the living environment for a disease. It allows the approach's results to be useable twice, by the patient's entourage and the building expert community.

\subsection{Formalisation of expert knowledge}

In addition to the challenges described earlier, another one exists linked to the expert knowledge, which needs to be gathered first, then validated and formalised afterwards. While International and European standards according to barrier-free living lack, the German DIN 18040-2 describe requirements even for wheelchair -accessible living that are well-structured and usable either. Relating to our proof of concept of a wheel-chaired, the expert $\mathrm{KB}$ only includes DIN criteria. Intending to expand our system to other common as well as non-common diseases e.g. Alzheimer's or Rheumatism, gathering similar valid criteria might be challenging and tedious. For this issue, we have already added the standardised Human Disease ontology (DO) in biomedical community by Schriml et al. (2019). DO has been developed collaboratively in order to provide consistent descriptions of human disease terms and facilitated discussion with experts in healthcare.

The absence of proper approaches for the evaluation of housing suitability under care-related aspects serves as a best practice study. We learned about several challenges that being tackled with a knowledge-based MDSS linked to BIM and using semantic technologies. The following chapter specifies more details of our methodology subdivided into our three main components, hereby state of the art technologies are used in an advantageous way.

\section{METHODOLOGY}

Our approach is based on knowledge and aims at providing a MDSS to assist aging in place by identifying the suitability of the living environment by rooms or furniture elements according to a patient's disease. It results appear in two different forms, in an IFC file representing a BIM model with the suitability of objects and an explanation of that classification as added value. The proposed approach takes 3D data acquired by state of the art acquisition techniques as input. The methodology is composed of two main process steps using AI, a knowledge-based data processing and a knowledge-based decision support. Figure 1 presents an overview of the methodology with its main steps and its associated processing actions. The two main steps use expert knowledge to provide interchangeability, flexibility and adaptability to the system. Therefore, this section presents both, the proposed approach's knowledge modeling component and the two main processing steps of the methodology.

\subsection{Knowledge base modeling}

The proposed approach builds on a KB gathers its expert input from two different domains. First, object detection ontology including building object modeling and second, assisted living information for patients according to their disease. The latter comprises human disease terms from DO and knowledge of building object requirements for diseases from DIN 18040-2. To link these fields, the KB accounts for three local modules. In the following, the role and the global structure of each module is described.

Object detection ontology: This ontology for object detection originates from KnowDIP by Ponciano et al. (2019c) that provides flexibility and adaptability to different contexts and objects for the knowledge-based data processing regarding object detection. This approach uses a knowledge model gathering object, data and algorithm description, explained below.

Objects are described through their geometries (e.g. shape, orientation, surface) and features (e.g. color, texture, roughness). They are contained in data and belong to a scene. A scene is characterized through a context (e.g. indoor, outdoor) and describes topological relationships between objects. Data are described through features (e.g. density, resolution, size) and acquisition technology that generates it. They represent a scene and contain objects and geometries. The algorithm description relates to data and objects. An algorithm generates data and detects objects. It is defined as suitable for geometry and has data prerequisites and parameters. Its parameters are defined according to data and the object's features. 
Disease modeling: The KB component has integrated the $\mathrm{DO}$ by Schriml et al. (2019). This ontology contains all diseases vocabulary structured in different levels of disease sub-types such as disease by an infectious agent or disease of mental health e.g. cognitive disorder and sleep disorder. Diseases are described through an ID, a name, a description, synonyms, subsets, and references.

Modeling of building object requirements relating to disease: This role is based on the building object from data processing modeling and disease modeling. It aims primarily at evaluating the suitability of rooms and furniture objects within, but can also determine issues that need to adapt to fulfil suitability. To represent this expert knowledge, we have used rules allowing a reasoning on the object and disease profile for the decision making. These rules point at defining three states for rooms and objects inside according to a disease such as isSuitable, Need2Adapt and isNotSuitable. While isSuitable meaning that the room or object fills the requirements for a disease, isNotSuitable means non adaptable opposite. Need2Adapt means that the room or object does not fill the disease requirements yet but can be adapted to be suitable then.

With respect to that states, there are two groups of rules: rules concerning objects inside a room and rules concerning the room itself. Object-related rules define the suitability and need of adaptation of an object for a disease according to its type, its features (e.g. dimension) and its components (e.g. door handle). The following example illustrated the reasoning of our knowledge: a room with a window is not suitable for a wheelchaired if the window handle is higher than $1.05 \mathrm{~m}$. This expert knowledge defined in DIN 18040-2 allows us to define the logical rule presented in Listing 1 to assess the windows that are not suitable for a wheel-chaired.

\footnotetext{
$k d: W i n d o w(? w)^{\wedge} k d: R o o m(? r)^{\wedge} k d: i s I n s i d e(? w, ? r)^{\wedge}$ $k d:$ hasHandle $(? w, ? h)^{\wedge} k d: h a s D i s t a n c e D o u b l e(? h, ? d h)^{\wedge}$ $k d$ :Disease(?di)^kd:hasDistanceDouble(?di,?dwc)^ swrlb:greaterThan(?dh, ?dwc) -> kd:isNotSuitableFor(?w, ?di)
}

Listing 1. Logical rule defining the unsuitability of a window for a wheel-chaired. KnowDIP namespace is shortened by kd.

Rules concerning rooms define a room's state from above according to objects contained inside and the type of itself. There are three main global rules to define the state of a room: rule 1 refers to suitable rooms, rule 2 to unsuitable rooms and rule 3 to rooms needing adaptation. A room is suitable only if all objects inside got the state isSuitable. A room is unsuitable, if in it exists at least one object, which has the state isNotSuitable as presented in Listing 2. Finally, a room needs adaptation, if it contains no object with the state isNotSuitable and at least one object with the state Need2Adapt.

$k d: R o o m(? r)^{\wedge} k d: i$ Inside(?element, ?r $)^{\wedge} k d:$ Disease $(? d)^{\wedge}$ $k d$ :isNotSuitableFor(?element, ?d) -> kd:isNotSuitableFor(?r, ?d)

Listing 2. Logical rule defining the unsuitability of a room for any disease. KnowDIP namespace is shortened by kd.

\subsection{Knowledge-based data processing}

The processing of point cloud data provided as input is performed through the KnowDIP approach. This approach is based on two major components, which are the knowledge and a set of algorithms. These two components communicate together through SPARQL queries (Prud'hommeaux and Seaborne 2008) in order to (a) allow the knowledge to guide data processing by executing an algorithm, which has been selected and defined as executable and (b) allow the algorithm part to enrich the knowledge through a representation of the algorithm execution result. This knowledge-based approach of data processing has shown its performance and ability of adaptation to different contexts of application in previous works (Ponciano et al. 2019a), whose building indoor context (Ponciano et al. 2019b). It processes the point cloud data iteratively through three main steps: segmentation, classification, and a self-learning process (Ponciano 2019). The approach achieves the segmentation by using its knowledge on objects it needs to recognize, data to process, and available algorithms to select suitable methods for the case study. It then determines the order of algorithm execution according to knowledge on algorithm requirements and results of previously executed algorithms iteratively. The classification process is also performed thanks to knowledge. The OWL2 (Hitzler et al. 2009) formulisation describing objects creates the possibility of classifying a segment that results from algorithm execution by applying logical reasoning. Finally, the knowledge-based approach performs a self-learning process to tackle the limits of the knowledge description. This self-learning process uses the classification result to make hypotheses to improve object descriptions and thus their detection. This approach, based on AI, provides a flexible object detection and results in a KB containing the different objects, their features and their topological relationships in the scene are represented inside the input data (c.f. processing action 1 in Figure 1).

\subsection{Knowledge-based decision support}

The knowledge-based decision support process intends to provide a visualization and explanation of each object state. It firstly produces an annotated point cloud with colored objects according to their states and then give an explanation of that state (c.f. processing action 3 in Figure 1). This explanation contains both, a list of rooms with their corresponding states and a description of objects inside with their associated states that can serve as a checklist for unknowledgeable users. The output of the proposed approach is generated from the KB component content after having applied reasoning on it (c.f. processing action 2 in Figure 1). This reasoning process consists of applying expert rules, explained in section 3.1 on the object modeling obtained from the previous step and represented into the KB. Enriched with the several object states, the use of reasoning on a $\mathrm{KB}$ for decision making provides comprehensible and explainable results. Thus, this approach of decision support brings more transparency than a majority of DSS based on a black box system. Moreover, an IFC file is generated from the annotated point cloud by a manual process (c.f. processing action 4 in Figure 1). As a major benefit this 3D BIM model contains full building information combined with the inferred object states by geometric intersection. Thanks to that our results provide a detailed visualisation for patient's entourage and building expert community.

\section{PROOF OF CONCEPT}

The suggested approach presented in the previous section has been tested as part of a proof of concept (PoC). The approach has thus been applied on a point cloud representing one investigation room, described in subsection 4.1. Our PoC focuses on the walking disability of a wheel-chaired person. Expert rules related to building object constraints for a wheelchair are presented in subsection 4.2. Then, results for each step of the methodology are given and graphically illustrated in subsection 4.3 and 4.4 such as the result of data processing and the result of the decision support system. 


\subsection{Input point cloud of the case study}

For our PoC the S3DIS dataset by Armeni et al. (2016) is used. Out of this point cloud, we randomly selected a room and defined it as the area of investigation. As illustrated in Figure 2a, the raw point cloud is roughly sparse with few occlusions. Despite this, constitute room structures and furniture elements can be detected. The dataset represents several chairs, a large table in the middle of the room and a small cupboard towards the opened door. Besides, there is a special ceiling construction and a window at the opposite side. Light elements are also available, but are not considered in our approach.

\subsection{Expert knowledge modeling related to a wheel-chaired}

Rules applied for the PoC have been formulised from the requirements described in the German DIN 18040-2 with respect to a standard wheelchair of $0.70 \times 1.20 \mathrm{~m}$ related to barrier-free living and unrestricted wheelchair usage. In addition to the three global rules concerning rooms (c.f. section 3.1) 21 rules related to objects and specific to a wheel-chaired have been added to the KB. These rules represent the following assertions:

\begin{tabular}{|l|l|}
\hline & A room is not suitable for a wheel-chaired, if \\
\hline R1 & the floor has any kind of stairs or thresholds above $0.02 \mathrm{~m}$. \\
\hline R2 & $\begin{array}{l}\text { there are less than } 1.50 \times 1.50 \mathrm{~m} \text { free space zones for direction } \\
\text { changes and manoeuvring. }\end{array}$ \\
\hline R3 & $\begin{array}{l}\text { there are unmovable objects like tables, cupboards that limit } \\
\text { the free space zones for movements. }\end{array}$ \\
\hline R4 & there is no free parking space of at least $1.80 \times 1.50 \mathrm{~m}$. \\
\hline R5 & $\begin{array}{l}\text { there is less than } 1.20 \mathrm{~m} \text { space for movement in viewing } \\
\text { direction and } 1.50 \mathrm{~m} \text { across. }\end{array}$ \\
\hline R6 & areas are more sloped than $3 \%$. \\
\hline R7 & handles are turning handles or recessed handles. \\
\hline R8 & $\begin{array}{l}\text { the area in front of a door in opening direction is less than } \\
1.50 \times 1.50 \mathrm{~m} .\end{array}$ \\
\hline R9 & $\begin{array}{l}\text { the area in front of a door in reverse opening direction is less } \\
\text { than } 1.20 \times 1.50 \mathrm{~m} .\end{array}$ \\
\hline R10 & handrails are lower than $0.85 \mathrm{~m}$ or higher than $0.90 \mathrm{~m}$ \\
\hline R11 & there exist sharp edges. \\
\hline & A room with a door is not suitable for a wheel-chaired, if \\
\hline R12 & the door opening direction is into the room. \\
\hline R13 & the door handle is lower than $0.85 \mathrm{~m}$ or higher than $1.05 \mathrm{~m}$. \\
\hline R14 & the door width is lower than $0.90 \mathrm{~m}$. \\
\hline R15 & the door height is lower than $2.05 \mathrm{~m}$. \\
\hline & A room with a window is not suitable for a wheel-chaired, if \\
\hline R16 & the window handle is lower than $0.85 \mathrm{~m}$ or higher than $1.05 \mathrm{~m}$. \\
\hline R17 & the path to a window is blocked at a distance at $\geq 1.00 \mathrm{~m}$. \\
\hline R18 & the window opening direction is outside the room. \\
\hline R19 & the window height above ground is more than $0.60 \mathrm{~m}$. \\
\hline R20 & $\begin{array}{l}\text { A room with a table is not suitable for a wheel-chaired, if the } \\
\text { table surface is lower than } 0.80 \mathrm{~m} .\end{array}$ \\
\hline R21 & $\begin{array}{l}\text { A room with a cupboard is not suitable for a wheel-chaired, } \\
\text { if it is higher than } 1.30 \mathrm{~m} .\end{array}$ \\
\hline
\end{tabular}

Table 1. Rules that refer to a barrier-free living and unrestricted wheelchair usage for our case study.

Concerning the rule R11, we have defined a sharp edge as an edge having an angle under $90^{\circ}$.

\subsection{Results of knowledge-based data processing}

As explained in section 3.2, data processing is composed of data segmentation followed by an initial classification, which is then improved by a self-learning process. Figure 2 shows a visual representation of the input point cloud (a) and the results obtained after these processing steps (b and c). The segmentation step is performed by a region growing algorithm. This algorithm groups nearby points in the same segment if they have a similar normal vector. This algorithm is automatically configured by the KnowDIP framework ${ }^{2}$ according to the objects to be segmented, here mainly planar objects, and the data features such as noise or density.

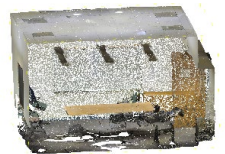

(a)

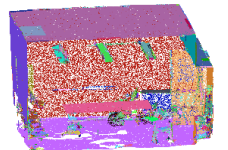

(b)

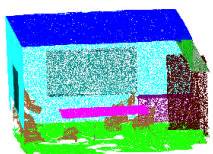

(c)
Figure 2. Overview of data processing step results: input point cloud (a), segmentation result (b), object detection result (c).

The result of this segmentation step, illustrated in Figure 2b, is a list of segments from which the proposed features such as dimension, planarity, location and connectivity are extracted. Each segment and its features are then integrated into the KB. After this integration, the reasoning on object modeling classifies these segments. The results of this classification are used as a learning base for self-learning, which adapts the object modeling to the specific context of the application case. Adapting the object modeling provides a better classification. The final result is shown in Figure 2c.

\subsection{Results of the decision support}

As explained in section 3.3 of the methodology, the decision support result depends on a rule-based reasoning. Table 2 presents the validation result for the 21 rules defined for the case study (c.f. section 4.2).

\begin{tabular}{|c|c|c|}
\hline & validated & invalidated \\
\hline R1 & & $\times$ \\
\hline R2 & $\times$ & \\
\hline R3 & & $\times$ \\
\hline R4 & & $\times$ \\
\hline R5 & $\times$ & \\
\hline R6 & & $\times$ \\
\hline R7 & - & - \\
\hline R8 & & $\times$ \\
\hline R9 & - & - \\
\hline R10 & & $\times$ \\
\hline R11 & & $\times$ \\
\hline
\end{tabular}

\begin{tabular}{|c|c|c|}
\hline & validated & invalidated \\
\hline R12 & $\times$ & \\
\hline R13 & $\times$ & \\
\hline R14 & $\times$ & \\
\hline R15 & $\times$ & \\
\hline R16 & $\times$ & \\
\hline R17 & & $\times$ \\
\hline R18 & - & - \\
\hline R19 & $\times$ & \\
\hline R20 & $\times$ & \\
\hline R21 & & $\times$ \\
\hline & & \\
\hline
\end{tabular}

Table 2. Validation result of the case study. Hereby,

invalidated means there is no information available to validate the rule. Empty fields indicate no test candidates.

From the rule validation results, the adapted state has been added to each object inside the room. The result of data processing has recognised the door and rule-based reasoning has identified its dimensions do not fit the requirements. The door has a height of $2.01 \mathrm{~m}$, which validates the rule $\mathrm{R} 15$ and a width of $0.82 \mathrm{~m}$, which validates the rule R14. Furthermore, the door being open during the acquisition process, so the data processing has allowed to identify its opening direction as into the room, which validates the rule R12. The door also has a handle, at a height of $1.05 \mathrm{~m}$ and thus validates the rule R13. With the validation of rules R12-R15, the door obtains the isNotSuitable state. The window has a handle that is higher than the maximum height for a wheelchaired and validates the rule R16. The window also has an above ground height superior to $0.60 \mathrm{~m}$, which validates the rule R19. Thus, the window obtains the isNotSuitable state too. The table has a height of $0.7 \mathrm{~m}$, which validates the rule R20. Therefore, the table obtains the isNotSuitable state. Finally, the free space of the

\footnotetext{
https://github.com/JJponciano/knowdip
} 
room is not sufficient according to the rule $R 2$. The free space is defined according to movable and unmovable objects and was calculated as the difference of the room's area to the total sum of the area occupied by several objects. If the sum of the area occupied by unmovable objects does not provide sufficient free space, then these objects are considered as not suitable in terms of rule R3. In this case, the area occupied by the table and cupboard objects allow sufficient free space. After estimating the free space according to the unmovable objects, the same process is carried out with the movable objects such as the remaining chairs. In this example, the total sum of the chairs also allows sufficient free space. However, the chair's current arrangement does not provide a continuous area of $1.50 \times 1.50 \mathrm{~m}$ free space concerning rule R2. Because of the movable chair objects that can be arranged differently the rule R2 assigns the Need2Adapt state to these chairs. The validation of rule R5 is similar to the validation of the rule $\mathrm{R} 2$, with a change in dimension in the computation of free space zones (c.f. Table 1). It also obtains a similar result.

The application of the rule set let's our MDSS define the state of each object according to the disease profile and produces a point cloud annotated with the defined states. This annotated point cloud is then used to enrich the IFC file illustrated in Figure 3a with those states as finally shown in Figure $3 \mathrm{~b}$. Red objects represent the isNotSuitable state, while green objects mean isSuitable. Furthermore, orange objects are relating to the Need2Adapt state and different colors represent other objects, which have no checkable requirements for a wheelchair usage in terms of barrier-free living.

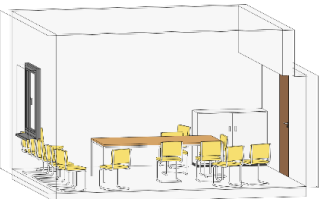

(a)

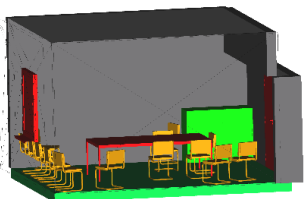

(b)
Figure 3. BIM of the room of investigation: IFC model (a) and result of the reasoning process of our MDSS transferred to IFC model (b).

In addition to the point cloud and the IFC file annotated with object state, our approach generates an explanation of inferred results. Results presented in Figure $3 \mathrm{~b}$ illustrates the following suitability and the need of adaptation from the objects of the proof of concept room. Table 3 refers to the justifications of the decisions made.

\begin{tabular}{|l|l|}
\hline Window is unsuitable & $\begin{array}{l}\text { due to its handle that is too high. } \\
\text { due to its height above ground that is } \\
\text { higher than } 0.60 \mathrm{~m} .\end{array}$ \\
\hline Door is unsuitable & $\begin{array}{l}\text { due to an incorrect opening direction. } \\
\text { due to its handle that is too high. } \\
\text { due to its width that is too narrow. } \\
\text { due to its height that is too low. }\end{array}$ \\
\hline Table is unsuitable & due to its height that it too low. \\
\hline Chairs need to be adapted & due to insufficient free space. \\
\hline Floor is suitable & \\
\hline Cupboard is suitable & $\begin{array}{l}\text { due to unsuitable window, door and } \\
\text { table. }\end{array}$ \\
\hline Room is unsuitable & \\
\hline
\end{tabular}

Table 3. Explanation result of the reasoning process of our medical decision support system.

\section{CONCLUSION AND FUTURE WORK}

With our research, we take into account demands and wishes of an increasingly aging society to live a self-determined life in their own houses as long as possible without dangerous restrictions.
The aim of this research is to develop a transparent decision support system as part of an emerging E-Health technology supporting aging in place by evaluating living sites. Subjects of the evaluation are in particular constitute and furniture objects like walls, tables, chairs, which have been processed in terms of an individual disease and thus classified as suitable or unsuitable for living at home without professional caregivers. For this purpose, we used different expert knowledge such as (a) human health and (b) building construction into our knowledge base. Thanks to that we are able to process $3 \mathrm{D}$ point cloud data, which describe as-is living sites in a detailed manner and thus delivers a great information resource automatically. As a result of the knowledge-based reasoning after data acquisition we produce an annotated point cloud illustrating objects that are suitable for the disease, unsuitable or need adaption towards being suitable afterwards. This annotation is transferred later into BIM paradigm to achieve more precise visualisation and description in building context. We have tested and validated our proposed approach in a case study according to the walking disability of a wheel-chair bound person successfully. Thanks to the transparent processing methodology and the comprehensible output through BIM, we expect to reduce scepticism of the elderly towards new technology, as our objective is to support patients on the one hand and to unburden caregivers on the other.

In future works we will take into consideration both, a more realistic use case with further disease profiles and the automatic generation of BIM components through ifcOWL proposed by Pauwels and Terkaj (2016). The latter promises to increase our level of automation by adding more knowledge from construction domain through IFC schema, which provide the entire information of BIM components. In addition, an automated BIM generation process empowers further developments linked to IoT technology aiming intelligent guidance and interaction with elderly or residents in general. We will also address the textual explanation in order to provide a transparent understanding of our OWL results.

\section{ACKNOWLEDGEMENTS}

This study is part of an ongoing Ph.D. research at i3mainz. The authors thank Antoine Hafsaoui for proofreading this article.

\section{REFERENCES}

Adan, A.; Huber, D. (2011): 3D Reconstruction of Interior Wall Surfaces under Occlusion and Clutter. In: 2011 International Conference on 3D Imaging, Modeling, Processing, Visualization and Transmission (3DIMPVT). Hangzhou, TBD, China, 16.05.2011 19.05.2011: IEEE, pp. 275-281.

Armeni, I.; Sener, O.; Zamir, A. R.; Jiang, H.; Brilakis, I.; Fischer, M.; Savarese, S. (2016): 3D Semantic Parsing of Large-Scale Indoor Spaces. In: IEEE Conference on Computer, pp. 1534-1543.

Augustyniak, P. (2013): Adaptive architecture for assisted living systems. In: 6th International Conference on Human System Interactions (HSI). Sopot, Poland, 06.06.2013 - 08.06.2013: IEEE, pp. 562-569.

Avila, N.; Sampogna, C. (2011): e-Health - Ambient Assisted Living and Personal Health Systems. In: Handbook of Medical Technology. Springer Handbooks: Berlin Heidelberg.

Baig, M. M.; GholamHosseini, H.; Moqeem, A. A.; Mirza, F.; Lindén, M. (2019): Clinical decision support systems in hospital care using ubiquitous devices: Current issues and challenges. In: Health informatics journal 25 (3), pp. 1091-1104. DOI: $10.1177 / 1460458217740722$. 
Banbury, A.; Roots, A.; Nancarrow, S. (2014): Rapid review of applications of e-health and remote monitoring for rural residents. In: The Australian journal of rural health 22 (5), pp. 211-222. DOI: 10.1111/ajr.12127.

Bassier, M.; Mattheuwsen, L.; Vergauwen, M. (2019): BIM Reconstruction: Automated Procedural Modeling from Point Cloud Data. In: Int. Arch. Photogramm. Remote Sens. Spatial Inf. Sci. XLII2/W17, pp. 53-60. DOI: 10.5194/isprs-archives-XLII-2-W17-532019.

Bassier, M.; Yousefzadeh, M.; van Genechten, B. (2015): Evaluation of data acquisition techniques and workflows for Scan to BIM. In: Geo Business.

Baumgärtel, D.; Mielke, C.; Haux, R. (2018): A Review of Decision Support Systems for Smart Homes in the Health Care System. In: Stud Health Technol Inform. 247, pp. 476-480. DOI: 10.3233/9781-61499-852-5-476.

Ben-Shabat, Y.; Avraham, T.; Lindenbaum, M.; Fischer, A. (2018): Graph based over-segmentation methods for 3D point clouds. In: Computer Vision and Image Understanding 174, pp. 12-23. DOI: 10.1016/j.cviu.2018.06.004

Billis, A. S.; Papageorgiou, E. I.; Frantzidis, C. A.; Tsatali, M. S.; Tsolaki, A. C.; Bamidis, P. D. (2015): A decision-support framework for promoting independent living and ageing well. In: IEEE journal of biomedical and health informatics 19 (1), pp. 199-209. DOI: 10.1109/JBHI.2014.2336757.

Borrmann, A.; König, M.; Koch, C.; Beetz, J. (2015): Building Information Modeling. Wiesbaden: Springer Fachmedien Wiesbaden.

Bouchard, B.; Giroux, S.; Bouzouane, A. (2007): A Keyhole Plan Recognition Model for Alzheimer's Patients: First Results. In: Applied Artificial Intelligence 21 (7), pp. 623-658. DOI: 10.1080/08839510701492579.

Chang, A. X.; Funkhouser, T.; Guibas, L.; Hanrahan, P.; Huang, Q.; Li, Z.; Savarese, S.; Savva, M.; Song, S.; Su, H.; Xiao, J.; Yi, L.; Yu, F. (2015): ShapeNet: An Information-Rich 3D Model Repository. Available online at http://arxiv.org/pdf/1512.03012v1.

Chen, J.; Kira, Z.; Cho, Y. K. (2019): Deep Learning Approach to Point Cloud Scene Understanding for Automated Scan to 3D Reconstruction. In: J. Comput. Civ. Eng. 33 (4), p. 4019027. DOI: 10.1061/(ASCE)CP.1943-5487.0000842.

Chiang, T.-C.; Liang, W.-H. (2015): A Context-Aware Interactive Health Care System Based on Ontology and Fuzzy Inference. In: Journal of medical systems 39 (9), p. 105. DOI: 10.1007/s10916015-0287-2.

Coelho, F.; Relvas, S.; Barbosa-Póvoa, A. P. (2021): Simulationbased decision support tool for in-house logistics: the basis for a digital twin. In: Computers \& Industrial Engineering 153, p. 107094. DOI: $10.1016 /$ j.cie.2020.107094.

Dietenbeck, T.; Torkhani, F.; Othmani, A.; Attene, M.; Favreau, J.M. (2017): Multi-layer Ontologies for Integrated 3D Shape Segmentation and Annotation. In: Advances in Knowledge Discovery and Management, vol. 665. Cham: Springer International Publishing (Studies in Computational Intelligence), pp. 181-206.

DIN 18040-2:2011-09, Barrierefreies Bauen - Planungsgrundlagen Teil 2: Wohnungen.

Dutta, G.; Gupta, N.; Fourer, R. (2011): An optimization-based decision support system for strategic planning in a process industry: the case of aluminium company in India. In: Journal of the Operational Research Society 62 (4), pp.616-626. DOI: 10.1057/jors.2010.8.
Eastman, C. M. (2008): BIM handbook. A guide to building information modeling for owners, managers, designers, engineers, and contractors. Hoboken N.J.: Wiley.

European Commission (2020): European Commission Report on the Impact of Demographic Change. Available online at https://ec.europa.eu/info/sites/info/files/demography_report_2020_n .pdf, checked on 1/22/2021.

Fiannaca, A.; Gaglio, S.; La Rosa, M.; Peri, D.; Rizzo, R.; Urso, A. (2011): A Knowledge Based Decision Support System for Bioinformatics and System Biology. In: Computational Intelligence Methods for Bioinformatics and Biostatistics, vol. 6685. Berlin, Heidelberg: Springer Berlin Heidelberg (Lecture Notes in Computer Science), pp. 215-228.

Harrefors, C.; Sävenstedt, S.; Axelsson, K. (2009): Elderly people's perceptions of how they want to be cared for: an interview study with healthy elderly couples in Northern Sweden. In: Scandinavian journal of caring sciences 23 (2), pp. 353-360. DOI: 10.1111/j.14716712.2008.00629.x

Hitzler, P.; Krötzsch, M.; Parsia, B.; Patel-Schneider, P. F.; Rudolph, S. (2009): OWL 2 Web Ontology Language Primer. W3C Recommendation. Available online at https://www.w3.org/TR/owl2primer/.

Hussain, M.; Ali, T.; Khan, W. A.; Afzal, M.; Lee, S.; Latif, K. (2015): Recommendations service for chronic disease patient in multimodel sensors home environment. In: Telemedicine journal and e-health: the official journal of the American Telemedicine Association 21 (3), pp. 185-199. DOI: 10.1089/tmj.2014.0028.

ISO 16739-1:2018-11, Industry Foundation Classes (IFC) for data sharing in the construction and facility management industries - Part 1: Data schema.

Jung, J.; Hong, S.; Yoon, S.; Kim, J.; Heo, J. (2016): Automated 3D Wireframe Modeling of Indoor Structures from Point Clouds Using Constrained Least-Squares Adjustment for As-Built BIM. In: $J$. Comput. Civ. Eng. 30 (4). DOI: 10.1061/(ASCE)CP.19435487.0000556 .

Kaczmarek, M.; Ruminski, J.; Bujnowski, A. (2011): Multimodal platform for continuous monitoring of elderly and disabled at home. In: (FedCSIS) Federated Conference on Computer Science and Information Systems, pp. 393-400.

Kihlgren, A.; Svensson, F.; Lövbrand, C.; Gifford, M.; Adolfsson, A. (2016): A Decision support system (DSS) for municipal nurses encountering health deterioration among older people. In: $B M C$ nursing 15, p. 63. DOI: 10.1186/s12912-016-0184-0.

Kumar, D. S.; Sathyadevi, G.; Seelan, S. (2011): Decision Support System for Medical Diagnosis Using Data Mining. In: (IJCSI) International Journal of Computer Science Issues 8 (3).

Lehtola, V. V.; Kaartinen, H.; Nüchter, A.; Kaijaluoto, R.; Kukko, A.; Litkey, P.; Honkavaara, E.; Rosnell, T.; Vaaja, M. T.; Virtanen, J.-P.; Kurkela, M.; El Issaoui, A.; Zhu, L.; Jaakkola, A.; Hyyppä, J. (2017): Comparison of the Selected State-Of-The-Art 3D Indoor Scanning and Point Cloud Generation Methods. In: Remote Sensing 9 (8), p. 796. DOI: 10.3390/rs9080796.

Lercari, N. (2016): Terrestrial Laser Scanning in the Age of Sensing. In: Digital Methods and Remote Sensing in Archaeology. Cham: Springer International Publishing (Quantitative Methods in the Humanities and Social Sciences), pp. 3-33.

Leung, K. H.; Choy, K. L.; Siu, P. K. Y.; Ho, G. T. S.; Lam, H. Y.; Lee, C. K. M. (2018): A B2C e-commerce intelligent system for reengineering the e-order fulfilment process. In: Expert Systems with Applications 91, pp. 386-401. DOI: 10.1016/j.eswa.2017.09.026. 
Lin, C.-C.; Tai, Y.-C.; Lee, J.-J.; Chen, Y.-S. (2017): A novel point cloud registration using 2D image features. In: EURASIP J. Adv. Signal Process. 2017 (1). DOI: 10.1186/s13634-016-0435-y.

Lopez-Guede, J. M.; Moreno-Fernandez-de-Leceta, A.; MartinezGarcia, A.; Graña, M. (2015): Lynx: Automatic Elderly Behavior Prediction in Home Telecare. In: BioMed research international 2015, p. 201939. DOI: $10.1155 / 2015 / 201939$.

Lu, Q.; Lee, S. (2017): Image-Based Technologies for Constructing As-Is Building Information Models for Existing Buildings. In: $J$. Comput. Civ. Eng. 31 (4). DOI: 10.1061/(ASCE)CP.19435487.0000652 .

Malinverni, E. S.; Pierdicca, R.; Paolanti, M.; Martini, M.; Morbidoni, C.; Matrone, F.; Lingua, A. (2019): Deep Learning for Semantic Segmentation of 3D Point Cloud. In: Int. Arch. Photogramm. Remote Sens. Spatial Inf. Sci. XLII-2/W15, pp. 735742. DOI: 10.5194/isprs-archives-XLII-2-W15-735-2019.

Manar, J.; Mouna, B.; Naima, A. M.; Samy, H.; Zineb, S.; Mohammed, B. O. (2017): Evaluation of the Decision Support Systems. In: JCC 14 (3). DOI: 10.17265/1548-7709/2017.03.004.

Marschollek, M. (2012): Decision support at home (DS@HOME) system architectures and requirements. In: BMC medical informatics and decision making 12, p. 43. DOI: 10.1186/1472-6947-12-43.

Masaro, A.; Mustich, A.; Galiano, A. (2020): Decision Support System for Multistore Online Sales Based on Priority Rules and Data Mining. In: csit 8 (1). DOI: 10.13189/csit.2020.080101.

Ohta, R.; Ryu, Y.; Kitayuguchi, J.; Gomi, T.; Katsube, T. (2020): Challenges and solutions in the continuity of home care for rural older people: A thematic analysis. In: Home health care services quarterly 39, pp. 126-139. DOI: 10.1080/01621424.2020.1739185.

Papathanasiou, J.; Ploskas, N.; Linden, I. (2016): Real-World Decision Support Systems. Cham: Springer International Publishing (37).

Papathoma-Köhle, M.; Cristofari, G.; Wenk, M.; Fuchs, S. (2019): The importance of indicator weights for vulnerability indices and implications for decision making in disaster management. In: International Journal of Disaster Risk Reduction 36, p. 101103. DOI: 10.1016/j.ijdrr.2019.101103.

Pauwels, P.; Terkaj, W. (2016): EXPRESS to OWL for construction industry: Towards a recommendable and usable ifcOWL ontology. In: Automation in Construction 63, pp. 100-133. DOI: 10.1016/j.autcon.2015.12.003.

Ponciano, J.-J.; Karmacharya, A.; Wefers, S.; Atorf, P.; Boochs, F. (2019a): Connected Semantic Concepts as a Base for Optimal Recording and Computer-Based Modelling of Cultural Heritage Objects. In: Structural Analysis of Historical Constructions, vol. 18. Cham: Springer International Publishing (RILEM Bookseries), pp. 297-304.

Ponciano, J.-J. (2019): Object detection in unstructured 3D data sets using explicit semantics. Dissertation. Université de Saint-Étienne, Saint-Etienne.

Ponciano, J.-J.; Boochs, F.; Trémeau, A. (2017): Knowledge-based object recognition in point clouds and image data sets. In: gis.Science - Die Zeitschrift für Geoinformatik. Available online at https://hal.archives-ouvertes.fr/hal-02047375.

Ponciano, J.-J.; Boochs, F.; Trémeau, A. (2019b): 3D object recognition through a process based on semantics and consideration of the context. In: Oldenburger 3D Tage. Oldenburg. Available online at https://hal.archives-ouvertes.fr/hal-02651917.

Ponciano, J.-J.; Trémeau, A.; Boochs, F. (2019c): Automatic Detection of Objects in 3D Point Clouds Based on Exclusively Semantic Guided Processes. In: IJGI 8 (10), p. 442. DOI: 10.3390/ijgi8100442.
Poux, F.; Billen, R. (2019): Voxel-based 3D Point Cloud Semantic Segmentation: Unsupervised Geometric and Relationship Featuring vs Deep Learning Methods. In: IJGI 8 (5), p. 213. DOI: 10.3390/ijgi8050213.

Poux, F.; Neuville, R.; Nys, G.-A.; Billen, R. (2018): 3D Point Cloud Semantic Modelling: Integrated Framework for Indoor Spaces and Furniture. In: Remote Sensing 10 (9), p. 1412. DOI: 10.3390/rs10091412.

Poux, F.; Ponciano, J.-J. (2020): Self-Learning Ontology for Instance Segmentation of 3D Indoor Point Cloud. In: Int. Arch. Photogramm. Remote Sens. Spatial Inf. Sci. XLIII-B2-2020, pp. 309-316. DOI: 10.5194/isprs-archives-XLIII-B2-2020-309-2020.

Prud'hommeaux, E.; Seaborne, A. (2008): SPARQL Query Language for RDF. W3C Recommendation. Available online at https://www.w3.org/TR/rdf-sparql-query/, checked on 1/29/2021.

Qi, C. R.; Su, H.; Mo, K.; Guibas, L. J. (2016): PointNet: Deep Learning on Point Sets for 3D Classification and Segmentation. Available online at http://arxiv.org/pdf/1612.00593v2.

Richter, J.; Vogel, S. (2020): Illustration of Clinical Decision Support System Development Complexity. In: Studies in Health Technology and Informatics 272, pp. 261-264. DOI: $10.3233 /$ SHTI200544.

Rösler, U.; Schmidt, K.; Merda, M.; Melzer, M. (2018): Digitalisierung in der Pflege. Wie intelligente Technologien die Arbeit professionell Pflegender verändern. Bundesanstalt für Arbeitsschutz und Arbeitsmedizin. Berlin.

Schriml, L. M.; Mitraka, E.; Munro, J.; Tauber, B.; Schor, M.; Nickle, L.; Felix, V.; Jeng, L.; Bearer, C.; Lichenstein, R.; Bisordi, K.; Campion, N.; Hyman, B.; Kurland, D.; Oates, C. P.; Kibbey, S.; Sreekumar, P.; Le, C.; Giglio, M.; Greene, C. (2019): Human Disease Ontology 2018 update: classification, content and workflow expansion. In: Nucleic acids research 47 (D1), D955-D962. DOI: 10.1093/nar/gky1032.

Simões-Marques, M. (2016): SINGRAR-A Distributed Expert System for Emergency Management: Implementation and Validation. In: Real-World Decision Support Systems, vol. 37. Cham: Springer International Publishing (37), pp. 275-302.

Stark, B.; Knahl, C.; Aydin, M.; Elish, K. (2019): A Literature Review on Medicine Recommender Systems. In: International Journal of Advanced Computer Science and Applications (IJACSA) $10(8)$.

Stula, S. (2012): Living in Old Age in Europe - Current Developments and Challenges. Working Paper No. 7 of the Observatory for Sociopolitical Developments in Europe. Available online at http://www.sociopolitical-observatory.eu/uploads/tx aebgppublications/AP_7_EN.pdf, checked on 1/19/2021.

Suphavilai, C.; Bertrand, D.; Nagarajan, N. (2018): Predicting Cancer Drug Response using a Recommender System. In: Bioinformatics (Oxford, England) 34 (22), pp. 3907-3914. DOI: 10.1093/bioinformatics/bty452.

Sutton, R. T.; Pincock, D.; Baumgart, D. C.; Sadowski, D. C.; Fedorak, R. N.; Kroeker, K. I. (2020): An overview of clinical decision support systems: benefits, risks, and strategies for success. In: NPJ digital medicine 3, p. 17. DOI: 10.1038/s41746-020-0221-y.

Wiles, J. L.; Leibing, A.; Guberman, N.; Reeve, J.; Allen, R. E. S. (2012): The meaning of "aging in place" to older people. In: The Gerontologist 52 (3), pp. 357-366. DOI: 10.1093/geront/gnr098.

Wu, Z.; Song, S.; Khosla, A.; Yu, F.; Zhang, L.; Tang, X.; Xiao, J. (2015): 3D ShapeNets: A deep representation for volumetric shapes. In: 2015 IEEE Conference on Computer Vision and Pattern Recognition (CVPR). Boston, MA, USA, 07.06.2015 - 12.06.2015: IEEE, pp. 1912-1920. 